\title{
Segmentation of complex images based on component-trees: Methodological tools
}

\author{
Benoît Caldairou $^{(\mathrm{a}, \mathrm{c}, \mathrm{d})}$, Benoît Naegel ${ }^{(\mathrm{b})}$, Nicolas Passat ${ }^{(\mathrm{a})}$ \\ (a) Université de Strasbourg, LSIIT, UMR CNRS 7005, France \\ (b) Université Nancy 1, LORIA, UMR CNRS 7503, France \\ (c) Université de Strasbourg, LINC, UMR CNRS 7191, France \\ (d) École Supérieure Chimie Physique Électronique de Lyon, France \\ e-mail: \{benoit.caldairou,passat\}@unistra.fr, benoit.naegel@loria.fr
}

\begin{abstract}
Component-trees can be used for the design of image processing methods, and in particular segmentation ones. However, despite their ability to consider various kinds of knowledge and their tractable computation, methodological deadlocks still forbid to efficiently involve them in real applications. In this article, we explore new solutions to some of these deadlocks, and more especially those related to $(i)$ complexity of the structures of interest and (ii) multiple knowledge handling. The usefulness of the proposed strategies is illustrated by preliminary results related to vessel segmentation from 3-D angiographic data.
\end{abstract}

Key words: Component-trees, segmentation, attribute-filtering, grey-level images.

\section{Introduction}

The component-tree (also known as dendrone [5,3], confinement tree [7] or max-tree [14]) is a graph-based structure which models some characteristics of a grey-level image by considering its binary level-sets obtained from successive thresholding operations.

Initially proposed in the field of statistics, the component-tree has been (re)defined in the theoretical framework of mathematical morphology and involved, in particular, in the development of morphological operators $[2,14,13]$. Thanks to efforts devoted to its efficient computation $[2,14,7,11,8]$ or its use in complex knowledge handling procedures [15], component-trees have been considered for the design of various kinds of grey-level image processing methods, including image filtering and segmentation [5, $6,17,12,16,10]$, video segmentation [14], image registration [7], image compression [14], or image retrieval $[9,1]$.

Despite the ability of component-trees to consider complex/multiple knowledge and their tractable computation, methodological deadlocks still forbid to efficiently involve them in real applications. In this article, we propose to explore solutions to some of these deadlocks, and more especially those related to $(i)$ complexity of the (shape of) structures of interest and (ii) multiple knowledge handling.

In Section 2, previous works involving component-trees in the design of segmentation methods are described, emphasising the remaining challenges to be faced. Section 3 introduces definitions and notations required to make the article self-contained. In 
Section 4, some methodological considerations provide solutions to tackle the challenges stated in Section 2. An application, described in Section 5 for 3-D angiographic image segmentation illustrates the soundness of the proposed framework. Section 6 summarises the contributions of this article and points out the main perspectives.

\section{Segmentation based on component-trees}

As mentioned above, component-trees have been considered for the development of image segmentation methods, mainly in the field of (bio)medical imaging, and in particular for: dermatological data [10], wood micrographs [6], cerebral MRI [4], CT/MR angiography [19], or confocal microscopy [12].

It has to be noticed that their use is often only devoted to one specific step of the segmentation (marker selection in [4]), or to perform filtering [19,12], i.e. to remove "useless" parts of the processed image, leading to a superset of an actual segmentation. Among the methods which fully use component-trees for segmentation purpose, some can consider complex (i.e. multiple) knowledge [10] or can be run without userinteraction [6], but none of them is able to determine the correct pieces of knowledge required to perform segmentation without guidance of the user. Moreover, such methods only deal with simple-shape objects (circular or elliptical 2-D features in [6,10]).

This emphasises the fact that automatic segmentation of complex objects based on the use of multiple elements of knowledge obviously remains an open methodological problem in the field of component-tree-based methods, a fortiori when such knowledge also needs to be automatically determined (which may be necessary whenever the size of the parameter space becomes too large). In the next sections, we explore some ways to deal with this difficult issue. In particular, we consider strategies enabling to decrease the potential complexity of the structures of interest, and to determine the nodes (and thus the attributes) of the component-trees of ground truth images, then enabling automatic learning of correct parameters for segmentation purpose.

\section{Definitions and notations}

Let $n \in \mathbb{N}^{*}$. In the sequel, $[a . . b]$ (with $a, b \in \mathbb{Z}$ ) denotes the discrete interval $[a, b] \cap \mathbb{Z}$. We set $\overline{\mathbb{Z}}=\mathbb{Z} \cup\{-\infty\}$. A discrete grey-level image can be defined as a function $I: \mathbb{Z}^{n} \rightarrow \overline{\mathbb{Z}}$. The support of $I$ is defined by $\operatorname{supp}(I)=\left\{x \in \mathbb{Z}^{n} \mid I(x) \neq-\infty\right\}$. We assume that for any considered image $I, \operatorname{supp}(I)$ is finite. We will note $\operatorname{supp}(I)=E$ and $V=[a . . b] \subset \mathbb{Z}$, where $a=\min \{I(x) \mid x \in E\}$ and $b=\max \{I(x) \mid x \in E\}$. From now on, we will assimilate an image $I: \mathbb{Z}^{n} \rightarrow \overline{\mathbb{Z}}$ to its (finite) restriction $I_{\mid E}: E \rightarrow V$.

Let $X \subseteq E$. The connected components of $X$ are the equivalence classes of $X$ w.r.t. the equivalence relation on $E$ induced by the adjacency relation chosen for $\mathbb{Z}^{n}$. The set of the connected components of $X$ is noted $C[X]$.

Let $v \in V$. We set $\mathcal{P}(E)=\{X \mid X \subseteq E\}$. Let $X_{v}: V^{E} \rightarrow \mathcal{P}(E)$ be the thresholding function defined by $X_{v}(I)=\{x \in E \mid v \leq I(x)\}$ for all $I: E \rightarrow V$.

Let $v \in V$ and $X \subseteq E$. We define the cylinder function $C_{X, v}: E \rightarrow \overline{\mathbb{Z}}$ by $C_{X, v}(x)=v$ if $x \in X$ and $-\infty$ otherwise. A discrete image $I: E \rightarrow V$ can then be expressed as 
$I=\bigvee_{v \in V} C_{X_{v}(I), v}=\bigvee_{v \in V} \bigvee_{X \in C\left[X_{v}(I)\right]} C_{X, v}$, where $\bigvee$ is the pointwise supremum for the sets of functions.

Let $\mathcal{K}=\bigcup_{v \in V} C\left[X_{v}(I)\right]$. The relation $\subseteq$ is a partial order on $\mathcal{K}$, and the Hasse diagram $(\mathcal{K}, L)$ of the partially ordered set $(\mathcal{K}, \subseteq)$ is a tree (i.e. a connected acyclic graph), the root of which is the supremum $R=\sup (\mathcal{K}, \subseteq)=E$. This rooted tree $(\mathcal{K}, L, R)$ is called the component-tree of $I$. The elements $\mathcal{K}, R$ and $L$ are the set of the nodes, the root and the set of the edges of the tree, respectively.

Note that each node of $\mathcal{K}$ is a binary connected component distinct from all the other ones. However, such a connected component can be an element of $C\left[X_{v}(I)\right]$ for several (successive) values $v \in V$. For each $X \in \mathcal{K}$, we set $m(X)=\max \{v \in V \mid$ $\left.X \in C\left[X_{v}(I)\right]\right\}=\min _{x \in X}\{I(x)\}$. An image $I: E \rightarrow V$ can then be defined from its component-tree $(\mathcal{K}, L, R)$ as $I=\bigvee_{X \in \mathcal{K}} C_{X, m(X)}$.

Component-trees enable the storage - at each node - of elements of information, also called attributes, related to the binary connected component associated to the node. It is possible to consider any kind of quantitative/qualitative and scalar/vectorial attributes, provided they can be conveniently formalised. Pruning a component-tree $(\mathcal{K}, L, R)$ of an image $I$ according to the attributes stored at the nodes (by removing the nodes having a non-correct attribute w.r.t. a given criterion) enables to perform filtering on $I$. The filtered image $I_{f}$ is then defined as $I_{f}=\bigvee_{X \in \mathcal{K}_{f}} C_{X, m(X)}$ where $\mathcal{K}_{f} \subseteq \mathcal{K}$ is the subset of the remaining nodes after the pruning process. When performing segmentation, a binary result $I_{b}$ can similarly be obtained as $I_{b}=\bigcup_{X \in \mathcal{K}_{f}} X$.

\section{Methodological concepts}

In this section, we present methodological tools enabling to develop algorithms based on component-trees, and dealing with the main challenges described in Section 2. In Subsection 4.1, solutions are proposed to spatially decompose (and reconstruct) an image, thus breaking complex structures into (hopefully) simpler sub-ones. In Subsection 4.2, the way to automatically extract relevant nodes from the component-tree of a ground truth (i.e. a correctly segmented) image is discussed, enabling to avoid userinteraction in segmentation processes.

\subsection{Image partitioning/reconstruction}

The binary connected components at the nodes $X \in \mathcal{K}$ of a component-tree may possibly be complex and/or gather several structures of interest of the associated image. In such cases, these nodes, potentially composed of several semantic elements may be hard to detect/discriminate due to the heterogeneity of the characterising properties of these elements.

In order to illustrate this assertion, let us consider the grey-level image of Fig. 1(a), which is composed of four semantic entities: squares, disks, thin straight lines and thick curves. Here, we obtain a critical situation where only one - useless - node is available at each level of the tree, as observed in Fig. 1(b-e). Despite the existence of specific properties (elongation, straightness, compactness, etc.) for each kind of elements, their specific intensity in the image and/or their spatial organisation (connections, generation 


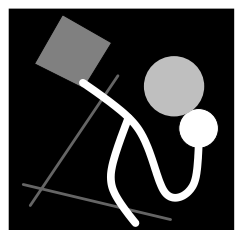

(a)

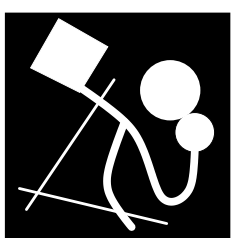

(b)

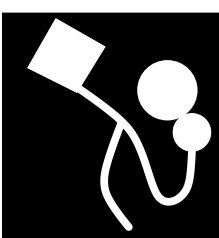

(c)

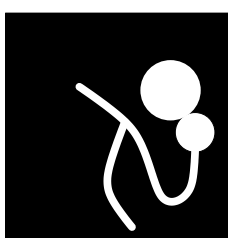

(d)

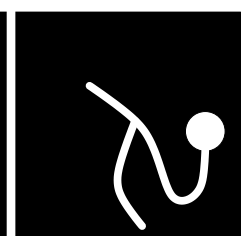

(e)

Fig. 1. (a) A grey-level image containing different semantic elements (geometric shapes). (be) Threshold images obtained from (a) at successive grey-levels: the obtained nodes/connected components do not enable to discriminate the visualised elements (see text).

of complex shapes from simpler ones, etc.) result in a component-tree the nodes of which do not enable any characterisation.

In similar cases, the computation of attributes devoted to characterise accurate and specific properties will generally fail. It has to be noticed that such situations are not infrequent in real applications. For instance, in angiographic image analysis (see Section 5), vessels are generally organised into a unique network, thus making attributes characterising single tubular structures inefficient.

A solution to this general issue can consist in processing the image as a collection of smaller subimages, hence enabling to split complex structures into smaller - and hopefully easier to detect - sub-ones. A straightforward strategy based on this approach is the following one.

1. Divide $I: E \rightarrow V$ into a set of images $I^{k}: E^{k} \rightarrow V(k \in[1 . . m])$ such that $\left\{E^{k}\right\}_{k=1}^{m}$ is a partition of $E$, and $I_{\mid E^{k}}=I^{k}$ for all $k \in[1 . . m]$.

2. Compute, for each $k \in[1 . . m]$, the component-tree of $I^{k}$ and perform segmentation, then generating a binary output image $B^{k} \subseteq E^{k}$.

3. Define the segmentation result $B$ by merging all the results $B^{k}: B=\bigcup_{k=1}^{m} B^{k}$.

This simple and potentially useful approach however suffers from two drawbacks: (i) the partition of $E$ may split a structure of interest between several subsets $E^{k}$, thus forbidding its correct detection, and (ii) the size of the subsets $E^{k}$, possibly well-chosen to fit a given structure, may be non-adapted to the detection of another one.

Partitioning A way to avoid these two drawbacks is to compute a redundant and multiscale decomposition of $I$, in order to fit at best the different structures of interest. The support $E$ of $I$ is then split by defining a set $\mathcal{E}_{\alpha, \beta}=\bigcup_{a \in \alpha}\left\{E_{a, \beta}^{k}\right\}_{k=1}^{m_{a, \beta}}$, such that for all $a \in \alpha$ we have ${ }^{1}$

$$
\forall k \in\left[1 . . m_{a, \beta}\right],\left|E_{a, \beta}^{k}\right|=|E| / a,
$$

\footnotetext{
${ }^{1}$ Note that in Eq. (2) the inclusion (instead of an equality) between the two elements implies that the set $\left\{E_{a, \beta}^{k}\right\}_{k=1}^{m_{a, \beta}}$ is actually not always a partition of $E$ since some of the $E_{a, \beta}^{k}$ may be partially out of $E$ to guarantee the same redundancy $\beta$ at each point of $E$.
} 


$$
\begin{array}{r}
E \subseteq \bigcup_{k=1}^{m_{a, \beta}} E_{a, \beta}^{k}, \\
\forall x \in E,\left|\left\{X \in\left\{E_{a, \beta}^{k}\right\}_{k=1}^{m_{a, \beta}} \mid x \in X\right\}\right|=\beta,
\end{array}
$$

where $\alpha \subseteq[1 . .|E|]$ is a set of volume ratios ("scales"), and $\beta \in \mathbb{N}^{*}$ is the "redundancy factor" of the pseudo-partitions $\left\{E_{a, \beta}^{k}\right\}_{k=1}^{m_{a, \beta}}$ at each scale $a \in \alpha$. It may generally be convenient to define $\alpha$ as a subset of $\left\{2^{n k}\right\}_{k \geq 0}$ in order to build subsets $E_{a, \beta}^{k}$ of $E \subset \mathbb{Z}^{n}$ in an "octree" fashion.

Reconstruction Once processed, each partial image $I_{a, \beta}^{k}: E_{a, \beta}^{k} \rightarrow V$ provides a binary output $B_{a, \beta}^{k} \subseteq E_{a, \beta}^{k}$. We set $\mathcal{B}_{\alpha, \beta}=\bigcup_{a \in \alpha}\left\{B_{a, \beta}^{k}\right\}_{k=1}^{m_{a, \beta}}$. By opposition to the initially proposed strategy, which enables to recover $B \subseteq E$ by simply merging the subimages $B^{k}$, the one proposed above does not straightforwardly lead to a final result, since overlaps induced by both multiscale and redundancy may lead to ambiguous results for any point $x \in E$ (depending on the image $I_{a, \beta}^{k}$ where $x$ is considered).

For any $x \in E$, let $\mathcal{E}_{\alpha, \beta}^{x}=\left\{E_{a, \beta}^{k} \in \mathcal{E}_{\alpha, \beta} \mid x \in E_{a, \beta}^{k}\right\}$ and $\mathcal{B}_{\alpha, \beta}^{x}=\left\{B_{a, \beta}^{k} \in \mathcal{B}_{\alpha, \beta} \mid x \in B_{a, \beta}^{k}\right\}$ (note that $0 \leq\left|\mathcal{B}_{\alpha, \beta}^{x}\right| \leq\left|\mathcal{E}_{\alpha, \beta}^{x}\right|=\beta$. $|\alpha|$ ). Final images $B_{f} \subseteq E$ and $I_{f}: E \rightarrow[0,1]$ (binary and fuzzy, respectively) can be reconstructed as follows

$$
\begin{aligned}
B_{f} & =\left\{x \in E|\lambda \leq| \mathcal{B}_{\alpha, \beta}^{x} \mid\right\} \text { for a given } \lambda \in[1, \beta .|\alpha|], \\
I_{f}(x) & =\left|\mathcal{B}_{\alpha, \beta}^{x}\right| /(\beta .|\alpha|) \text { for all } x \in E .
\end{aligned}
$$

It can be noticed that $(i)$ setting $\lambda=1$ in Eq. (4) is equivalent to define $B_{f}=\bigcup_{X \in \mathcal{B}_{\alpha, \beta}} X$, and (ii) $B_{f}$ can be obtained by thresholding $I_{f}$ at the considered value $\lambda$.

\subsection{Multiple criteria handling}

It is possible to involve arbitrarily large and heterogeneous sets of knowledge in segmentation processes by associating to each node of the component-tree vectorial attributes (containing qualitative, quantitative, structural information, etc.). This can lead to very accurate descriptions of the structures to be segmented. However, a straightforward and undesired side effect is the difficulty to determine, among the whole (and potentially huge) parameter space $\Omega$ induced by this knowledge, the correct subset $\omega \subset \Omega$ characterising the structures of interest, a fortiori in an interactive fashion.

In such conditions it becomes fundamental to enable automatic determination of such characterising subsets. This can be done by using learning - and in particular classification - tools. To this end, it is necessary to find a way to put in correspondence a "ground truth" (i.e. correct examples of what should be segmented) and the closest result which may be obtained by the component-tree-based method.

The problem to solve may be formalised as follows. Let $I_{g}: E \rightarrow V$ be a ground truth image (similar to those to be further processed by the method), and $B_{g} \subseteq E$ be the correct segmentation of this image. Let $(\mathcal{K}, L, R)$ be the component-tree of $I_{g}$. Let $\mathcal{S}=\left\{\cup_{X \in C} X\right\}_{C \subseteq \mathcal{K}}$ be the set of all binary images which can be generated from the set of nodes $\mathcal{K}$. In general, we will - unfortunately - never have $B_{g} \in \mathcal{S}$. We then 
need to determine the "best" binary image which may be computed from $\mathcal{K}$ w.r.t. $B_{g}$. This requires to define a (pseudo)distance $d$ on $\mathcal{P}(E)$ enabling to compare $B_{g}$ and the candidate binary images of $\mathcal{S}$. In particular, the best binary image $\hat{B}$ can be defined as

$$
\hat{B}=\arg \min _{B \in \mathcal{S}}\left\{d\left(B, B_{g}\right)\right\} .
$$

In this context, several strategies can reasonably be considered.

- By setting $d^{-}\left(B, B_{g}\right)=\left|B_{g} \backslash B\right|$ if $B \subseteq B_{g}$ and $+\infty$ otherwise, we have $\hat{B}^{-}=$ $\max _{\subseteq}\left\{B \in \mathcal{S} \mid B \subseteq B_{g}\right\}$, i.e. $\hat{B}^{-}$is the largest object included in $B_{g}$ which may be built from $\mathcal{K}$.

- By setting $d^{+}\left(B, B_{g}\right)=\left|B \backslash B_{g}\right|$ if $B \supseteq B_{g}$ and $+\infty$ otherwise, we have $\hat{B}^{+}=\min _{\subseteq}\{B \in$ $\left.\mathcal{S} \mid B_{g} \subseteq B\right\}$, i.e. $\hat{B}^{+}$is the smallest object including $B_{g}$ which may be built from $\mathcal{K}$.

The first (resp. second) strategy focuses on the elimination of false positives (resp. false negatives) with the side effect of possibly authorising the preservation of false negatives (resp. false positives). It has to be noticed that these asymmetric strategies can be efficiently implemented since the set of nodes generating $\hat{B}$ can obviously be computed with a (worst case) algorithmic complexity $O(|\mathcal{K}|)$ linear w.r.t. the number of nodes of the component-tree.

Some - more symmetric - strategies could also be proposed. The most straightforward one consists in setting $d^{*}\left(B, B_{g}\right)=\left|B_{g} \backslash B\right|+\left|B \backslash B_{g}\right|$. It aims at finding a "best compromise" between the amount of false positives and false negatives. In particular, we have $\hat{B}^{-} \subseteq \hat{B}^{*} \subseteq \hat{B}^{+}$. Also note that if $B_{g} \in \mathcal{S}$, we have $\hat{B}^{-}=\hat{B}^{*}=\hat{B}^{+}$. Surprisingly, this approach (by opposition to other symmetric ones, involving Hausdorff distances for example, which may present high algorithmic complexities) also leads to an algorithmic complexity $O(|\mathcal{K}|)$ (this claim will be proved in further works).

When a minimal set $\hat{B}$ has been extracted from $\mathcal{S}$, remains to determinate an adequate set of nodes $\hat{K} \subseteq \mathcal{K}$ associated to $\hat{B}$ (i.e. such that $\bigcup_{X \in \hat{K}} X=\hat{B}$ ). Let $\hat{C} \subseteq \mathcal{K}$ be the set defined by $\hat{C}=\{X \in \mathcal{K} \mid X \subseteq \hat{B}\}$ (note that the nodes of $\hat{C}$ generate a set of subtrees of the component-tree $(\mathcal{K}, L, R)$ of $\left.I_{g}\right)$. The set $\hat{B}$ can be generated by any set of nodes $\hat{K} \subseteq \hat{C}$ verifying $\bigcup_{X \in \hat{K}} X=\bigcup_{X \in \hat{C}} X=\hat{B}$. In order to determine such a set $\hat{K}$, two main strategies can, in particular, be considered.

- By setting $\hat{K}^{+}=\hat{C}$, any node included in $\hat{B}$ is considered as a useful (i.e. informative) binary connected component.

- By setting $\hat{K}^{-}=\{X \in \hat{C} \mid \forall Y \in \hat{C}, X \not \subset Y\}$, only the roots of the subtrees induced by $\hat{C}$ are considered as useful binary connected components.

The first (resp. second) strategy is the one considering the largest (resp. smallest) possible set of nodes/connected components among $\hat{C}$; in particular, it can be seen as the one which focuses at most on the grey-level (resp. binary) structure of the ground truth image $I_{g}$. The choice of the strategy may then be directed by the kind (binary $v s$. greylevel) of criteria/attributes to be considered.

Once a set of nodes $\hat{K}$ has been defined from the whole set $\mathcal{K}$ (from one or possibly several ground truth image(s)), the determination of the subset of characterising knowledge $\omega \subset \Omega$ has to be performed. Let $A: \mathcal{K} \rightarrow \Omega$ be the function associating, 


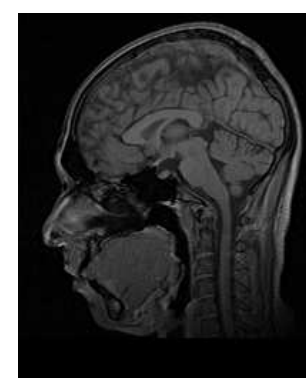

(a)

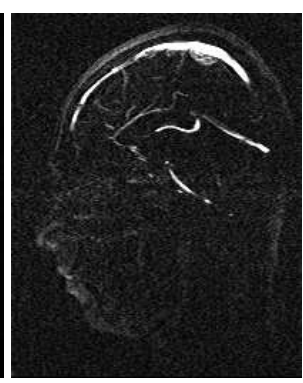

(b)

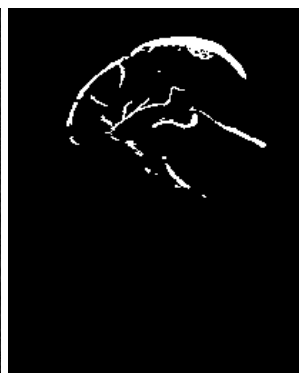

(c)

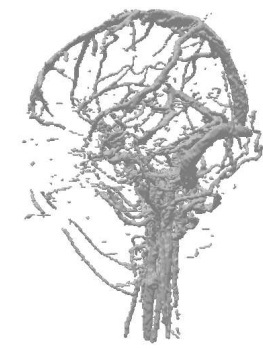

(d)

Fig. 2. (a,b) Phase contrast magnetic resonance angiography (ground truth image $I_{g}$ ): sagittal 2-D slices of the magnitude image $I_{m}$ (a) and of the phase image $I_{p}(\mathrm{~b})$. (c,d) Ground truth segmentation $B_{g}$ obtained from $I_{p}$ : sagittal slice (c) and 3-D visualisation (d).

to each node of a the component-tree, its stored attribute. The determination of $\omega$ can be expressed as a classification problem consisting in partitioning $\Omega$ into two classes thanks to the samples $A(\hat{K})=\{A(N) \mid N \in \hat{K}\}$ (corresponding to the attributes of the structures of interest) and $A(\mathcal{K} \backslash \hat{C})=\{A(N) \mid N \in \mathcal{K} \backslash \hat{C}\}$. This process can, for instance, be carried out by usual classification tools (such as the Support Vector Machine (SVM) [18], which has been considered in the experiments of the next section).

\section{A case study - Angiographic image segmentation}

Based on the framework described above, a strategy is being developed for segmenting 3-D angiographic data (namely phase contrast magnetic resonance angiographies - PCMRAs). We propose hereafter a preliminary and simplified description of this method, and we provide - for illustrative purpose - some obtained results. A complete description of the final method (with full validations and a larger set of involved attributes) will be found in dedicated further works.

PC-MRAs are bimodal images $\left(I_{m}, I_{p}\right) \in\left(V^{E}\right)^{2}$ where $I_{p}: E \rightarrow V$ is the phase (i.e. vascular) image while $I_{m}: E \rightarrow V$ is the magnitude (i.e. morphological) image, with $E=[0 . .255]^{3}$ and $V=[0 . . N] \subset \mathbb{N}$ (see Fig. 2(a,b)). The proposed method is devoted to segment phase images $I_{p}$ in order to extract the vessels (and in particular to discriminate them from noise and artifacts).

In order to enable a correct segmentation of the vessels from such images, three attributes have been considered: (i) the second Hu's moment, (ii) an inertia matrixbased elongation criterion, both computed from the component-tree of $I_{p}$, and (iii) the (signed) distance to the brain surface, computed from both $I_{m}$ (used for brain surface extraction) and the component-tree of $I_{p}$.

From these three attributes (generating a parameter space $\Omega \subset \mathbb{R}^{3}$ ), a vascular ground truth image $I_{g}$ (Fig. 2(a,b)) and its segmentation $B_{g}$ (Fig. 2(c,d)) have been involved in a learning process based on the computation of the best binary image $\hat{B}^{-}$ w.r.t. the $d^{-}$distance, and the computation of the corresponding set of nodes $\hat{K}^{-}$(the 


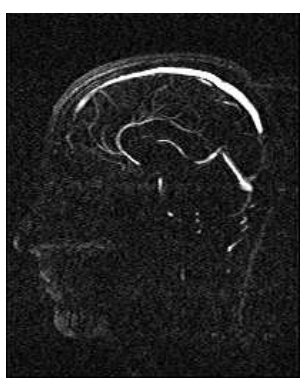

(a)

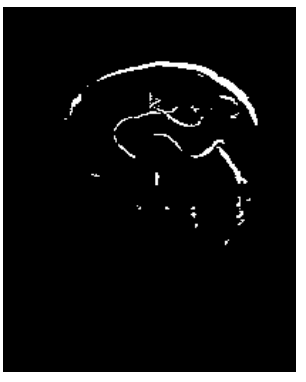

(b)

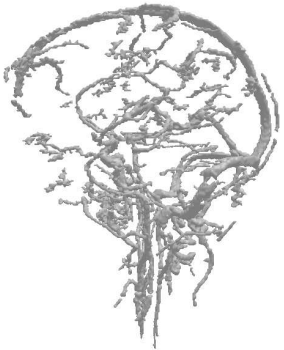

(c)

Fig. 3. (a) Phase contrast magnetic resonance angiography: sagittal 2-D slice of the phase image $I_{p}$. (b,c) Segmentation result (binary segmentation): sagittal 2-D slice (b) and 3-D visualisation (c).

choice of $d^{-} / \hat{B}^{-}$is linked to the considered ground truth data $I_{g}$ for which $B_{g}$ has been slightly oversegmented by the expert, while the choice of $\hat{K}^{-}$is the result of experimental considerations). An (automatic) SVM classification process has then been applied on the set of binary connected components of $\hat{K}^{-}$to determine an adequate set $\omega \subset \Omega$ of attribute values. PC-MRA phase images similar to $I_{g}$ have then been segmented in a multiscale fashion by using the attribute values of $\omega$. It has to be noticed that the segmentation process (and then the learning step) have been performed at several scales $(\alpha=\{1,8,64\})$, and with a redundancy factor $\beta=2$. The results have been obtained by fusing the partial binary images in a binary fashion (Eq. (4) with $\lambda=1$ ). An example of these results is illustrated in Fig. 3. It can been observed that, despite the presence of few false negatives, the obtained results globally present no artifacts (i.e. no false positive). This constitutes a satisfactory and encouraging property for the - difficult - analysis of such (non contrast-enhanced) data where vessels and artifacts present similar intensities and are often connected.

\section{Conclusion}

A generic framework, based on image partitioning and automatic selection of relevant structural elements from ground-truth data, has been proposed for the development of segmentation methods relying on component-trees. Methods based on this framework can automatically process complex images by use of potentially large sets of knowledge, as illustrated by an application devoted to 3-D angiographic data.

The concept of multiscale (i.e. spatial) decomposition has been explored. The decomposition of the image signal will also be considered in further works, leading to multiresolution approaches, permitting to enrich the proposed framework.

From an applicative point of view, a more complete version of the vessel segmentation method obtained from this framework, and introduced in Section 5 for illustrative purpose, will be described and fully validated in further works. 


\section{References}

1. N. Alajlan, M.S. Kamel, and G.H. Freeman. Geometry-based image retrieval in binary image databases. IEEE Transactions on Pattern Analysis and Machine Intelligence, 30(6):10031013, 2008.

2. E.J. Breen and R. Jones. Attribute openings, thinnings, and granulometries. Computer Vision and Image Understanding, 64(3):377-389, 1996.

3. L. Chen, M.W. Berry, and W.W. Hargrove. Using dendronal signatures for feature extraction and retrieval. International Journal of Imaging Systems and Technology, 11(4):243-253, 2000.

4. P. Dokládal, I. Bloch, M. Couprie, D. Ruijters, R. Urtasun, and L. Garnero. Topologically controlled segmentation of 3D magnetic resonance images of the head by using morphological operators. Pattern Recognition, 36(10):2463-2478, 2003.

5. P. Hanusse and P. Guillataud. Sémantique des images par analyse dendronique. In RFIA 1991, volume 2, pages 577-588, 1991.

6. R. Jones. Connected filtering and segmentation using component trees. Computer Vision and Image Understanding, 75(3):215-228, 1999.

7. J. Mattes and J. Demongeot. Efficient algorithms to implement the confinement tree. In DGCI 2000, volume 1953 of Lecture Notes in Computer Science, pages 392-405. Springer, 2000.

8. D. Menotti, L. Najman, and A. de Albuquerque Araújo. 1D component tree in linear time and space and its application to gray-level image multithresholding. In ISMM 2007, volume 1, pages 437-448. INPE, 2007.

9. V. Mosorov. A main stem concept for image matching. Pattern Recognition Letters, 26(8):1105-1117, 2005.

10. B. Naegel, N. Passat, N. Boch, and M. Kocher. Segmentation using vector-attribute filters: methodology and application to dermatological imaging. In ISMM 2007, volume 1, pages 239-250. INPE, 2007.

11. L. Najman and M. Couprie. Building the component tree in quasi-linear time. IEEE Transactions on Image Processing, 15(11):3531-3539, 2006.

12. G.K. Ouzounis and M.H.F. Wilkinson. Mask-based second-generation connectivity and attribute filters. IEEE Transactions on Pattern Analysis and Machine Intelligence, 29(6):9901004, 2007.

13. P. Salembier. Opérateurs connexes et arbres des coupes. In L. Najman and H. Talbot, editors, Morphologie Mathématique 1 : approches déterministes, chapter 5, pages 153-172. Hermès, 2008.

14. P. Salembier, A. Oliveras, and L. Garrido. Anti-extensive connected operators for image and sequence processing. IEEE Transactions on Image Processing, 7(4):555-570, 1998.

15. E.R. Urbach, N.J. Boersma, and M.H.F. Wilkinson. Vector attribute filters. In ISMM 2005, volume 30 of Computational Imaging and Vision, pages 95-104. Springer SBM, 2005.

16. E.R. Urbach, J.B.T.M. Roerdink, and M.H.F. Wilkinson. Connected shape-size pattern spectra for rotation and scale-invariant classification of gray-scale images. IEEE Transactions on Pattern Analysis and Machine Intelligence, 29(2):272-285, 2007.

17. E.R. Urbach and M.H.F. Wilkinson. Shape-only granulometries and gray-scale shape filters. In ISMM 2002, pages 305-314. CSIRO Publishing, 2002.

18. V. Vapnik. Statistical Learning Theory. Wiley-Interscience, New York, 1998.

19. M.H.F. Wilkinson and M.A. Westenberg. Shape preserving filament enhancement filtering. In MICCAI 2001, volume 2208 of Lecture Notes in Computer Science, pages 770-777. Springer, 2001. 